\title{
Definición de tres grupos de pacientes de enfermedad de Hodgkin según los niveles de Haptoglobina Sérica.
}

\author{
DRES.: GONZALEz B. *, NOSTE M. E. **, CARMONA A. $*$, DEL POZO, H. **, BeRESSI V. **, \\ MELENDEZ M. *. SORENSEN R. **.
}

INTRODUCCION. Las Haptoglobinas (Hp) son glicoproteínas responsable de la elevación de la fracción Alfa 2 globulina en el proteinograma en una serie de procesos inflamatorios y neoplásicos. Este aumento de las $\mathrm{Hp}$, junto a otras proteínas ubicadas en diferentes fracciones electroforéticas, como Alfa 1, constituye el denominado "Fenómeno de Fase Aguda" de la respuesta inmune inespecífica por parte del organismo.

El papel biológico de las Haptoglobinas no se conoce bien, aunque actualmente se sabe que se ligan sólidamente con la hemoglobina formando complejos que son eliminados de la circulación por el Sistema Retículo Endotelial. Su papel principal parece ser el impedir las pérdidas de hemoglobina y por ende del Fierro de la circulación (1).

Desde los trabajos de Jayle y col., se demostró el valor de las determinaciones de $\mathrm{Hp}$ en el suero de pacientes portadores de tumores, especialmente en la Enf., de Hodgkin. Estos autores comprobam ron que aquellos enfermos que tenían estos linfomas en etapa evolutiva presentaban un fuerte incremento de las tasas séricas de Hp y que éstas, bajo el efecto del tratamiento, volvían a sus valores normales, en estrecha correlación con la mejoría clínica del paciente $(2,3,4)$.

De esta manera, los niveles de Hp serían un reflejo más fidedigno de la actividad del proceso neoplásico que otros parámetros, como la velocidad de sedimentación, ya que en esta última, entran en juego variables que no influyen en la determinación de Hp.

El propósito de nuestro trabajo, fue el de analizar las concentraciones de $\mathrm{Hp}$ e Inmunoglo-

\footnotetext{
- Médico becado Hospital L. Calvo Mackenna.

* Depto. Inmunopatologia, Instituto Bacteriologico de Chile.

*n* Servicio de Oncologra, Hospital Calvo Mackenna.
}

bulinas en distintos tumores infantiles, especialmente en la Enf. de Hodgkin, relacionándolas con la actividad o remisión del paciente y con su posible correlación con el pronóstico de la enfermedad.

Material $Y$ método. Se analizaron 84 pacientes portadores de diversas neoplasias, controlados en el Servicio de Oncología del Hospital Luis Calvo Mackenna, desde Septiembre de 1969 hasta Mayo de 1973. Se descartaron aquellos enfermos que no fueron controlados durante un período superior de seis meses, tanto bajo el punto de vista clínico como de laboratorio.

Se incluyen en este estudio solamente aqueJlos pacientes a los cuales se les practicó tres determinaciones como mínimo de Hp.

Todo nuestro material lo constituyen enfermos en edad preescolar o escolar y cuyos diagnósticos fueron confirmados por una o más biopsias y demás exámenes complementarios. Agregamos a nuestro trabajo, cuatro niños con tumores benignos y 22 con adenopatías inflamatorias, tanto inespecíficas como específicas entre las cuales tuvimos algunas adenitis $T B C$, toxoplasmósicas $y$ otras. Como controles se analizaron a 100 sujetos sanos.

Las determinaciones de inmunoglobulinas y Haptoglobina se hicieron de acuerdo al método de inmunodifusión radial de Mancini, utilizando antisueros monoespecíficos en el Depto. de Inmunopatología del Instituto Bacteriológico.

Los valores normales de $\mathrm{Hp}$ en nuestra serie fueron de $198 \pm 57 \mathrm{mgr} \%$.

Resultados. En la Tabla Nọ 1 hemos analizado los valores promedios de $\mathbf{H p}$ en la Enf. de Hodgkin y otras neoplasias que están en trata- 
T A B L A 1

CONCENTRACIONES SERICAS DE HAPTOGLOBINA EN DIFERENTES NEOPLASIAS INFANTILES TRATADAS

\begin{tabular}{|c|c|c|c|c|c|c|c|}
\hline Grupos & Cusos & Muestras & Hpen mgr? & $D S$. & Rango & t.grupc & \\
\hline I. Enf. de Hodgkin & 23 & 250 & 339 & 202 & $37-832$ & I/Vl no signif. & $P>001$ \\
\hline II. Leucemias & 14 & 42 & 366 & 124 & $180-577$ & & \\
\hline \multicolumn{8}{|l|}{ IIl. Tumores sólidos } \\
\hline a) T. de Wilms & 12 & 96 & 232 & 101 & $1.35-449$ & a/V ns. & $P>001$ \\
\hline b) Neuroblastomas & 8 & 41 & 338 & $\llbracket 12$ & $127-479$ & b/V signific: & $p>005$ \\
\hline c) Linfosarcomas & 18 & 45 & 289 & 235 & 32.637 & c/V ns. & $p>001$ \\
\hline IV. Reticulosis & 9 & 26 & 285 & 91 & $149-469$ & & \\
\hline V. Tumores Benignos & 4 & 9 & 179 & 90 & $90-300$ & & \\
\hline VI. Adenopatías Inespecif. & 22 & 23 & 280 & 175 & $75-900$ & & \\
\hline VII. Controles. & 100 & 100 & 198 & 57 & $78-348$ & I/VII signific. & p> $>001$ \\
\hline
\end{tabular}

T A B I. A 2

NIVELES DE HP EN LA ENF. DE HODGKIN Y OTROS TUMORES ANTES Y DESPUES DEL TRATAMIENTO

\begin{tabular}{|c|c|c|c|c|c|c|}
\hline Grupos & Tratamieno & Casos & Muestras & Hp.en $m g r \%$ & t-grupos & \\
\hline \multirow[t]{2}{*}{ I. Enf. de Hodgkin } & a.- Antes & 18 & 18 & 373 & Ia/lb ns. & $\mathbf{p}>0,01$ \\
\hline & b.-Despuês & 18 & 169 & 335 & & \\
\hline \multirow[t]{2}{*}{ II. Tumores sólidos } & a.-Antes & 23 & 23 & 443 & IIa/llb signif. & $P>0,01$ \\
\hline & b.-Después & 23 & 129 & 252 & & \\
\hline \multirow[t]{2}{*}{ III. Leucemias } & a.-Antes & 5 & 5 & 316 & IIIa/lIIIb ns. & $\mathrm{p}>0,01$ \\
\hline & b.-Después & 5 & 20 & 230 & & \\
\hline
\end{tabular}

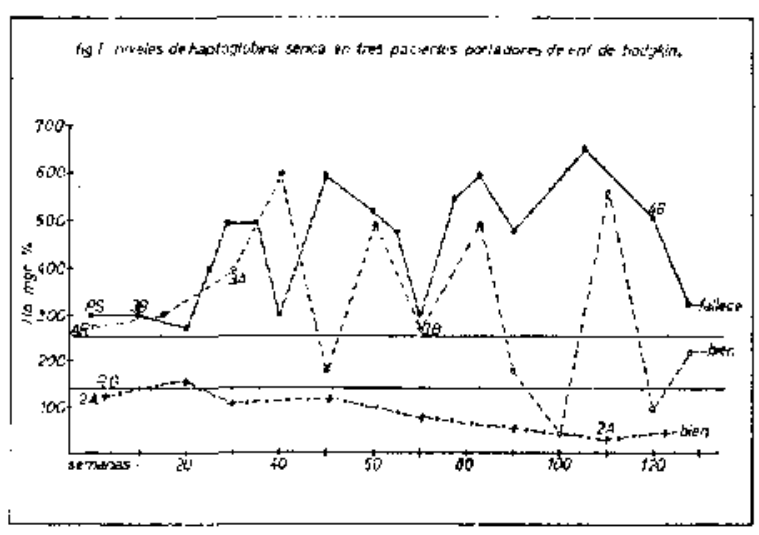

Figura No 1

miento comparándolas con los controles normales y los tumores benignos.

Se aprecia que no hubieron diferencias significativas entre los diversos tumores y entre éstos y las adenopatías. La diferencia es significativa cuando se compararon con los controles normales.

Los tumores benignos siempre mantuvieron cifras normales de $\mathrm{Hp}$ constatándose una diferen- cia a nivel de p menor que 0,05 con los Neuroblastomas y no así con los demás tumores sólidos cuyos valores no fucron estadísticamente significutivos.

En la Tabla No 2 se comparan los niveles de $\mathrm{Hp}$ en pacientes en períodos previos y posteriores al tratamicnto. Se aprecia una diferencia significativa sólo en los tumores sólidos.

Para poder interpretar estos resultados consideramos de importancia analizar como evolucionaban los niveles de $\mathrm{Hp}$ en nuestros enfermos portadores de Linfomas a lo largo del tratamiento.

Tala como se ve en la Fig. 1, éstos han seguido tres evoluciones totalmente diferentes. Un grupo, representado por el enfermo $P$. S., tuvo cifras permanentemente elevadas a pesar de un buen esquema terapéutico. De los 23 pacientes, hubo 9 que hicieron esta evolución. En cambio, 8 cnfermos hicieron una evolución clásica, descrita ampliamente, en la literatura, con cifras que fluctuaban con el tratamiento. Existe una elevación cuando el paciente experimenta una recidiva o aparición de una metástasis, y desciende cuando Ia enfermedad entra en remisión (paciente A. R.). En cambio, tuvimos 6 , en que las deter- 
T A B L A 3

NIYELES DE HP SEGUN EVOLUCION DE ESTA EN LA E. de HODGKIN

\begin{tabular}{lcccccc}
\hline Grupos & Casos & Muestras & Jpen mgr. $\%$ & Ds. & t-grupos \\
\hline Grupo I & 9 & 93 & 543 & 151 & I/II signif. & $\mathbf{p}<\mathbf{n 0 1}$ \\
Grupo II & 8 & 106 & 270 & 74 & II/III sjgnif. & $\mathbf{p}<001$ \\
Grupo III & 6 & 40 & 131 & 56 & I/III signif. & $\mathrm{p}<001$ \\
\hline
\end{tabular}

T A B L A 4

VALOR PRONOSTICO DE LA HP, EN LAA ENF. DE HODGKIN

\begin{tabular}{|c|c|c|c|}
\hline Grupos & No casos & Muertos & Vivos \\
\hline Grupo 1 & 9 & 7 & 2 \\
\hline Grupo 2 & 8 & 2 & 6 \\
\hline Grupo 3 & 6 & 1 & 5 \\
\hline
\end{tabular}

minaciones de $\mathrm{Hp}$ fueron siempre normales y que se representa por là evolución seguida por $\mathrm{cl}$ cnfermo R. G.

De esta manera nos interesó establecer tres grupos arbitrarios basados en estas diferentes evoluciones de la Hp. que denominamos grupos I, II, IJI respectivamente y que solamente reflejan el comportamiento de la $\mathrm{Hp}$ a través del tiempo.

En la Tabla No 3 , se aprecia como aguellos enfermos que mantuvieron niveles siempre elevados de $\mathrm{Hp}$ (Grupo $\mathrm{I}$ ) tienen un valor promedio de $543 \mathrm{mgr} \%$, a diferencia de los otros dos grupos, cuyas cifras son más bajas y con diferencials estadísticamente significativas respecto al grupo anterior,

En la Tabla No 4 consignamos para cada grupo cvolutivo de las $\mathrm{Hp}$ los pacientes fallecidos y los que aún sobreviven.

De 9 pacientes del grupo I, 7 fallecieron. De los 2 vivos, uno era portador adcmás de su linfoma, de una TBC ganglionar, lo que explica las cifras elevadas de Hp. El promedio de sobrevida de estos últimos, es de actualmente 29 meses, a diferencia de los fallecidos que tuvieron una sobrevida de solamente 17 meses.

En el grupo If, de un total de 8, seis pacientes viven en la actualidad con un promedio de sobrevida de 51 meses, y tenemos solamente 2 fallecidos. De estos que fallecieron, uno murió a consecuencía de una varicela después de 5 meses de tratamiento, y el otro, después de una evolución favorable de tres años y medio, bruscamente presentó una elevación de sus valores de Hp y posteriormente una metástasis pulmonar que lo llevó a la muerte.

Del grupo III, es decir, aquellos que tuvieron cifras permanentemente bajas de $\mathrm{Hp}$, de 6 pacientes hay 5 niños vivos, con un promedio de sobrevida de 23 meses y solamente un fallecido. Este último, durante el curso de su enfermedad presentó uná anemia hemolítica, lo que explica sus cifras bajas de Hp. Se suspendió el tratamiento esteroidal y falleció bruscamente. La necropsia reveló una meningitis purulenta residual, con focos de hemorragia suprarrenal y escasos elementos compatibles con un linfoma.

La correlación histopatológica utilizando la clasificación de Rye con la evolución de las Hp en los diversos grupos se ve en la Tabla No 5 , llamando la atención que a pesar que el número de pacientes es escaso, en el Grupo I hubo 4 niños de 9 en que la biopsia demostró una deplección linfocitaria.

En cambio, en el grupo III, sólo hay enfermos con predominio linfocítico y celularidad mixta $y$ ninguno con deplección linfocitaria.

En la Tabla No 6 destacamos la relación entro estos 3 grupos de $\mathrm{Hp}$ con los grados clínicos

T A B L A 5

CORRELACION ENTRE LA HISTOPATOLOGIA Y EVOLUCION DE LA HP EN LA EV DE HODGKIN

\begin{tabular}{lccc}
\hline Tipo Histológico & Grupo I & Grupo II & Grupo III \\
\hline Linfocítico Predominante & 0 & 0 & 2 \\
Celularidad Mfixta & 3 & 7 & 4 \\
Esclerosis Nodular & 2 & 1 & 0 \\
Deplexión Linfocitaria & 4 & 0 & 0 \\
\hline
\end{tabular}

T A B L A 6

RELACION ENTRE GRADOS CLINICOS Y GRUPOS DE HP. EN LA E. DE HODGKIN

Clasificación Clinica

Haptoglobina

1. Enfermedad Localizada 0032

2. Enf. ganglionar regional $0 \quad 2 \quad 2$

3. Enf. ganglionar bilateral $3 \quad 2 \quad 2$

4. Enf. generalizada 
de cada paciente, tomando corno base el último control practicado en el Servicio de Oncología. Existió una buena correlación entre el grupo I con los grados más avanzados de la enfermedad. Así, prácticamente todos los enfermos de este grupo, alcanzaron los grados 3 y 4 . Esta relación no es tan exacta para los otros dos grupos en que hubieron pacientes con diversos grados clínicos.

Así, en el grupo III, tuvimos 2 pacientes con grados clínico 3 , uno de los cuales falleció por una causa extratumoral $y$ el otro ha tenido una buena evolución. Asimismo, hay que hacer notar que varios de estos enfermos con grados avanzados y que tuvieron niveles de $\mathrm{Hp}$ permanentemente elevado, comenzaron con grados clínicos que hacían presumir una buena evolución.

Por último en la Fig. 2 estudiamos las concentraciones de Inmunoglobulinas (IgG e IgM) y $\mathrm{C}_{3}$ en aquellos enfermos que estaban en actividad clínica y en los que estaban en remisión. Se encontró en los primeros valores de $C_{3}$ en IgG algo más elevados que en los segundo. Aquellos pacientes con lesiones tumorales localizadas (grado I) siempre tuvieron $\mathrm{C}_{3}$ normales curante toda su evoluciôn a diferencia đe los grados más avanzados que en algunos se observó alza del $C_{3}$ en algún momento de su evolución. IgM.

No hubo diferencias significativas a nivel de

Discusion, De los resultados expuestos, se puede apreciar que los niveles de $\mathrm{Hp}$ no tienen valor como diagnóstico diferencial entre los diversos procesos malignos y tampoco con procesos inflamatorios. Sin embargo, debemos hacer notar que en estos áltimos, los niveles elevados de $\mathrm{Hp}$ tiender a normalizarse en un tiempo más corto que en las neeoplasias ya que su elevación se

Fig 2: Niveles deC3.IgG e IgM, en la E. de Hodgkin en relación a dctividad clínica.
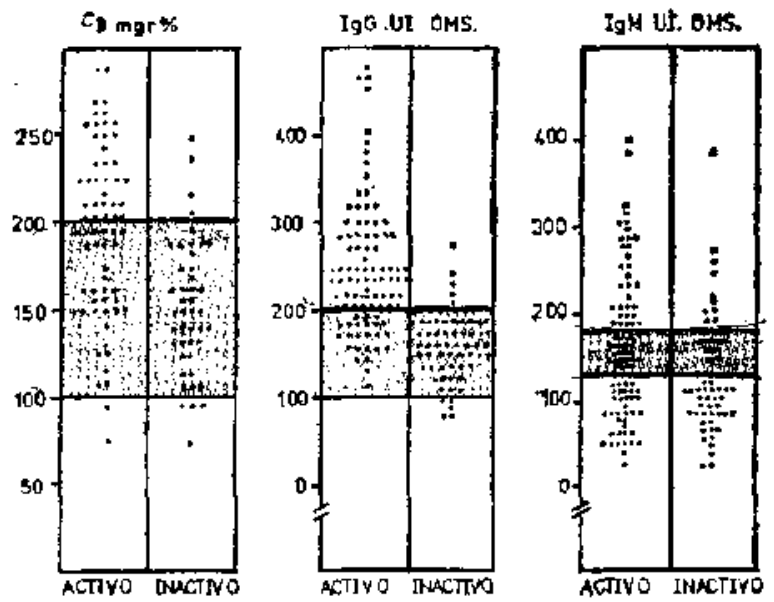

hace a expensas de la $\mathrm{Hp}$ que se encuentra distribuida en el espacio extravascular (5).

También hay que recalcar que todos los tumores benignos, aunque fueron escasos en nuestra casuística, tuvieron cifras dentro de rangos normales lo que podría ayudar en el diagnóstico diferencial con los tumores malignos. La determinación seriada de $\mathrm{Hp}$ es, a nuestro juicio, la única forma que permite evaluar bien la actividad o remisión de la enfermedad. Es así como algunos enfermos, estando en perfectas condiciones bajo el punto de vista clínico, han tenido elevaciones de sus niveles de Hp y posteriormente han aparecido metástasis o una reactivación del cuadro. Por esto, cuando existe una discrepancia entre ambos parámetros nos pone ante la obligación de practicar una reevaluación de nuestros enfermos.

En las leucemias nos llamó la atención en un comienzo que los niveles de Hp no fueron modificados con los tratamientos a que fueron sometidos los enfermos. Sin embargo, este mismo fenómeno ha sido descrito por otros autores como Peter Johan en cuya casuística sucede exactamente lo mismo cuando usa citostáticos y Prednisona, o esta última aisladamente. Cuando el citostático es dado en forma aislada la $\mathrm{Hp}$ desciende produciéndose una diferencia estadísticamente significativa (5).

Parece ser que los corticoides tiene un efecto estimulador de la síntesis de $\mathrm{Hp}$, por lo cual la mantención de niveles elevados no se debe a la falta de remisión de la enfermedad (7). De esta manera podríamos explicarnos cómo en los tumores sólidos hubo una diferencia apreciable de niveles de $\mathrm{Hp}$ bajo el efecto del tratamiento, ya que en estos casos se usan solamente citostáticos o radioterapia asociada a la cirugía.

Otro hecho significativo es la buena correlación entre los niveles de Hp con el pronóstico del paciente. De esta forma, aquellos enfermos que de partida mantienen concentraciones altas de Hp tienen muy mal pronóstico a pesar de catalogarlos clínicamente en grados no muy avanzados de la enfermedad.

Los estudios actuales concuerdan con que el aumento de esta glicoproteína se debe a un aumento de la síntesis a nivel hepático y no como se pensó antes, en una disminución del catabolismo secundario a un bloqueo del Sistema Retículo Endotelial (8).

En síntesis, en base a lo observado en nuestro trabajo pensamos que la determinación seriada de Hp aporta un elemento más en el estudio y tratamiento de los enfermos neoplásicos, especialmente en el control de la evolución y pronóstico de la enfermedad, teniendo sobre la velocidad de sedimentación, la ventaja de no ser influida por otros factores tales como la cantidad de fibrinógeno, el número de hematíes o proteínas de peso molecular elevado. 


\section{RESUMEN Y CONCLUSIONES}

1.- Los niveles de Hp no tienen valor como elemento diferencial entre los tumores y otros procesos inflamatorios. Tienen valor para diferenciar tumores benignos y malignos.

2.- La evolución de los niveles de $\mathrm{Hp}$ nos sirve para evaluar la actividad o remisión de la enfermedad, junto con otros elementos clinicos y de laboratorio.

3.- Niveles permanentemente elevados de $\mathrm{Hp}$, a pesar de un buen esquema terapéutico, nos permite definir a un grupo de enfermos en los cuales la enfermedad de partida es alta. mente maligna, independiente en algunos casos del cuadro clinico y/o histológico.

4.- - La determinación seriada de $\mathrm{Hp}$ es un examen sencillo de realizar y que debidamente interpretado ayuda a controlar la evolución de los pacientes con neoplasias.

\section{SUMMARY}

Haptoglobin levels were determined serially in chitdren with tumors and control groups. Analizing the results it is possible to draw the following conclusions:

1.- The levels of haptoglobin are of no value to differentiste between tumors and inflammatory processes. However they are of value in differentisting benign tumors.

2.- The follow-up of Hp levels, together with other laboratory values, allows us to evaluate the activity of the disease.
3.- The persistance of high levels of haptoglobin, despite a proper treatrent, is seen in a group of patients in which the disease is highly malignant. This sometimes is independent of clinical and hystological features.

4.- The determination of $\mathrm{Hp}$ values is easy to perform and helps in the control of the evolution of tumor patients.

\section{REFERENCIAS}

1.- Moe, P. S. Serum Haptoglobin level in disseminated malignant diseases in children. Acta Paediat. Scand., 59: 381-4, Jul. 70.

2.- Iayle, M. F. et al. Clinical value of haptoglobin and seromacoid determination in Hodgkin disease. Sem Hop. Paris, 44: 186t-7, Jun, 68.

3,-Kaplan, H. Hodgkin's disease, Cambridge, Mass. Harvard University Press, 1972.

4.-Kindler, $V$. Haptoglobin level in metastasizing tumors. Verh Dish. Ges. Fon. Med, 75: 524-6, 1969.

5.-Snyder, S. and Ashwell G. Quantitation of specific serum glycoproteins in malignancy. Clin. Chim. Acat., 34: 449-55, 1971.

6.-Galatius, F. The haptoglobins. A genetical study. Dansk Videnskabs Forlag, Kobenhayn, 1960.

7.-Joyle, $M$. Les seromneoides du sang: Jeur relations avec les mucoproteines de la substance fundamentale de tissue conjuntif Expos. Ann. Biochim. Med., 17: 757, 1955 . 